\title{
Proprioceptive regulation of voluntary ankle movements, demonstrated using muscle vibration, is impaired by Parkinson's disease
}

\author{
Etedal Khudados, Frederick W J Cody, Donald J O’Boyle
}

\begin{abstract}
Objective-To test the hypothesis that the proprioceptive regulation of voluntary movement is disturbed by Parkinson's disease, the effects of experimental stimulation of proprioceptors, using muscle vibration, on the trajectories of voluntary dorsiflexion movements of the ankle joint were compared between parkinsonian and control subjects.

Methods-Twenty one patients with Parkinson's disease, on routine medication (levodopa in all but one), and an equal number of age matched, neurologically intact controls, were trained initially to make reproducible ankle dorsiflexion movements $\left(20^{\circ}\right.$ amplitude with a velocity of $9.7^{\circ} / \mathrm{s}$ ) following a visual "go" cue while movement trajectories were recorded goniometrically. During $50 \%$ of the experimental trials, vibration $(105 \mathrm{~Hz} ; 0.7 \mathrm{~mm}$ peak to peak) was applied to the Achilles tendon during the ankle movement to stimulate antagonist muscle spindles; vibrated and non-vibrated trials were interspersed randomly. Subjects' performance was assessed by measuring end point position-that is, the ankle angle attained 2 seconds after the visual "go" cue, from averaged ( 20 trials) trajectories.
\end{abstract}

School of Biological Sciences

F W J Cody

Department of Psychology, University of Manchester, Manchester M13 9PL, UK

D J O'Boyle

Departments of Neurology and Surgery, Manchester Royal Infirmary, Manchester M13 9WL, UK

E Khudados

Correspondence to: Dr F W J Cody, School of Biological Sciences, Room 1.124, Stopford Building, University of Manchester, Manchester M13 9PT, UK. Telephone 004461275 5380; fax 0044612755363

Received 12 January 1999 and in final form

21 April 1999

Accepted 21 April 1999
Results-Statistical analysis of the end point amplitudes of movement showed that, whereas the amplitudes of nonvibrated movements did not differ significantly between patients with Parkinson's disease and controls, antagonist muscle vibration produced a highly significant reduction in the amplitudes of ankle dorsiflexion movements in both the patient and control groups. However, the extent of vibration induced undershooting produced in the patients with Parkinson's disease was significantly less than that in the controls; the mean vibrated/nonvibrated ratios were 0.86 and 0.54 for, respectively, the patient and control groups.

Conclusions-The present finding of a reduction of vibration induced ankle movement errors in parkinsonian patients resembles qualitatively previous observations of wrist movements, and suggests that Parkinson's disease may produce a general impairment of proprioceptive guidance.

(尹 Neurol Neurosurg Psychiatry 1999;67:504-510)
Keywords: Parkinson's disease; proprioception; vibration

Proprioceptive signals are generally thought to play a crucial part in normal human motor control. If the CNS is denied sensory inflow from the active limb-for example, by deafferentation ${ }^{1-3}$ - a profound impairment of the accuracy of all but the fastest, ballistic movements inevitably ensues. In addition, experimental stimulation of proprioceptors (for example, by muscle vibration), to interfere with the natural patterns of afferent discharge generated during willed movements, elicits kinaesthetic illusions $s^{45}$ and trajectory errors ${ }^{6-9}$ in healthy subjects.

Several authors ${ }^{10-12}$ have proposed that disturbances of proprioceptive regulation may underlie, or contribute to, the motor deficits which are characteristic of Parkinson's disease. An exaggeration of stretch reflexes, in particular their later components, in parkinsonian patients is well documented. ${ }^{13-15}$ In addition, patients with Parkinson's disease who have asymmetric signs overestimate the extent of movements made with the more affected limb when trying to make equivalent simultaneous movements of the two arms. ${ }^{16}$ Moore $^{16}$ interpreted his findings in terms of a mismatch of proprioceptive feedback and corollary discharge. Recently, Rickards and Cody ${ }^{17}$ have shown that vibration induced errors of voluntary wrist movements are significantly reduced in patients with Parkinson's disease, and concluded that this finding was consistent with an underlying disturbance of proprioceptive guidance.

In the experiments reported here, we studied the effects of muscle vibration on the kinematics of willed movements of the ankle in patients with Parkinson's disease and in control subjects for two main reasons. Firstly, such novel findings for movements of the ankle, in neurologically unimpaired subjects, would provide comparative data for those already available for wrist trajectories. Secondly, we sought to test the generality, across anatomically and functionally distinct joints, of pathophysiological disturbances of proprioceptive guidance in basal ganglia dysfunction. Brief details of this work have been reported previously in abstract form. ${ }^{18}$

\section{Methods}

SUBJECTS

Twenty one patients with idiopathic Parkinson's disease (15 men, six women; mean (SD) 
Clinical details of the parkinsonian patients investigated

\begin{tabular}{|c|c|c|c|c|c|}
\hline Patient & Age (y) & Sex & Duration (y) & Grading & Treatment \\
\hline 1 & 68 & M & 11 & 11 (II) & LD; Se \\
\hline 2 & 79 & M & 3 & 10 (II) & $\mathrm{LD} ; \mathrm{Se}$ \\
\hline 3 & 61 & $\mathrm{~F}$ & 3 & 5 (II) & LD; Se \\
\hline 4 & 50 & M & 10 & 11 (II) & LD; AC \\
\hline 5 & 63 & M & 5 & 14 (II) & LD \\
\hline 6 & 69 & M & 6 & 8 (II) & LD; Se \\
\hline 7 & 77 & M & 12 & 11 (II) & $\mathrm{LD} ; \mathrm{Se}$ \\
\hline 8 & 68 & M & 10 & 11 (II) & LD; AC; Amant \\
\hline 9 & 58 & $M$ & 12 & 8 (II) & LD; Se; AC \\
\hline 10 & 72 & M & 4 & 9 (II) & LD \\
\hline 11 & 66 & $\mathrm{~F}$ & 6 & 8 (II) & LD \\
\hline 12 & 62 & M & 6 & 7 (II) & $\mathrm{LD} ; \mathrm{Se}$ \\
\hline 13 & 59 & M & 15 & 11 (II) & LD; Perg \\
\hline 14 & 57 & $\mathrm{~F}$ & 9 & 7 (II) & LD; Perg \\
\hline 15 & 58 & M & 2 & 9 (II) & LD; Se; Perg \\
\hline 16 & 75 & $\mathrm{~F}$ & 2 & 7 (II) & LD \\
\hline 17 & 74 & $\mathrm{~F}$ & 6 & 12 (III) & LD; AC \\
\hline 18 & 67 & $\mathrm{~F}$ & 7 & 8 (II) & AC; Perg \\
\hline 19 & 55 & M & 12 & 12 (II) & LD; Se; AC \\
\hline 20 & 69 & M & 20 & 15 (II) & LD; AC; Perg \\
\hline 21 & 54 & M & 13 & 10 (II) & LD; AC; Amant; Perg \\
\hline mean & 64.8 & - & 8.3 & $9.7(-)$ & - \\
\hline SD & 8.1 & - & 4.7 & $2.5(-)$ & - \\
\hline
\end{tabular}

Grading refers to the Webster ${ }^{21}$ rating and, in parentheses, the Hoehn and Yahr $^{20}$ staging. Duration=Duration of Parkinson's disease since initial diagnosis; F=female; $M=$ male; $\mathrm{LD}=$ levodopa; $\mathrm{Se}=$ selegiline; $\mathrm{AC}=$ anticholinergic $;$ Amant $=$ amantadine; $\mathrm{Perg}=$ pergolide .

age 64.8 (8.1) years), according to the criteria of Hughes et al, ${ }^{19}$ and 21 neurologically unimpaired, control subjects (seven men, 14 women; mean (SD) age 63.3 (8.6) years) were studied. The controls were spouses or friends of the patients. Patients were studied while they were on their normal medication; their clinical details are shown in the table.

All subjects participated with fully informed consent and the protocols were approved by the Central Manchester Health Authority research ethics committee.

\section{EXPERIMENTAL PROCEDURES}

The subject sat comfortably in a chair with one leg supported in a frame which restricted movement to the ankle joint. During experimental trials, subjects were required to make voluntary dorsiflexion movements of the ankle. A precision goniometer (Penny and Giles, Ltd) was attached around the medial aspect of the ankle joint to record its movements. Subjects were denied direct vision of their ankle movements by a screen over the leg. A personal computer running purpose written software (Integrated Measurement Systems, Southampton, UK) controlled the presentation of auditory and visual cues to the subject.

Before the main series of experimental trials, subjects practised the movement task by visuomotor tracking. During this period, subjects viewed a visual display unit (VDU; subject's monitor). Two cursors on the screen displayed, respectively, the subject's movement trace and a computer generated target trace. During an initial practice period, the subject's task was to superimpose the movement cursor on the target cursor as the two cursors moved in temporal synchrony across the screen. The target trajectory was a dorsiflexion movement of the ankle joint of $20^{\circ}$ (from $120^{\circ}$ in plantar flexion to $100^{\circ}$ in dorsiflexion, corresponding to an upward screen deflection of the movement cursor) at a constant velocity of $9.7^{\circ} / \mathrm{s}$. For each practice movement, an auditory warning cue (time $=0 \mathrm{~s}$ ) sounded 1 second before the appearance of the cursors at the left hand edge of the VDU screen. For the initial period $(0.47 \mathrm{~s})$ of the sweep, the target cursor remained at its starting vertical level and no ankle movement was required. The target cursor then started to move upwards (visual "go" cue, time $=+1.47 \mathrm{~s}$ relative to auditory warning) and traced a ramp trajectory (vertical displacement of $20^{\circ}$ ) across the screen during the following 2.06 seconds, at the end of which it reached the right hand edge of the screen (time $=+3.53 \mathrm{~s})$. Subjects made a series $(\mathrm{n} \geqslant 15)$ of practice ankle dorsiflexion movements.

During the main series of experimental trials, the movement cursor was initially displayed at the left hand edge of the VDU screen to allow subjects to align the cursor with a marker indicating the desired starting position. Thereafter, the movement cursor was extinguished from the screen. The auditory warning signal and the VDU display of target waveform were retained to provide timing cues. Subjects were then required to reproduce the practised movement profile as accurately as possible, in the absence of visual feedback of movement performance. Voluntary dorsiflexion movements of the ankle were made either in the absence (non-vibrated, NV) or presence (vibrated, V) of vibration of the Achilles tendon.

Each subject performed a series of 40 dorsiflexion movements of the ankle, among which equal numbers of $\mathrm{NV}$ and $\mathrm{V}$ trials were interspersed in a pseudorandom manner. Shortly after the movement period had finished, subjects were instructed to relax the ankle musculature fully and, thereby, to allow the joint to fall under gravity to a neutral position. Subjects then returned their ankle to the desired starting angle, in readiness for the next trial, by visual reference to the movement cursor which reappeared at the right hand edge of the VDU screen. The intertrial interval was 5 seconds. This procedure minimised any clues to subjects concerning the occurrence and extent of any movement errors.

The more affected side was routinely studied in patients with Parkinson's disease, except where the disease was symmetric, when the dominant leg was used. The side of testing within the sample of neurologically intact subjects was allocated so that an identical proportion of control and parkinsonian subjects was studied on the dominant side.

\section{ANTAGONIST MUSCLE VIBRATION}

High frequency (105 Hz sinusoidal displacement of peak to peak amplitude $0.7 \mathrm{~mm}$ ) mechanical stimulation was applied transcutaneously to the Achilles tendon of the test ankle using a small, counterweighted and suspended, electromagnetic vibrator. ${ }^{17}$ The vibrator, which was left in contact with the tendon throughout, was activated during $\mathrm{V}$ trials and switched off during NV trials. During V trials, a 2 second train of vibration commenced 0.3 seconds (time $=+1.77 \mathrm{~s}$ relative to auditory warning) after the onset of the visual "go" cue (the start of the upward ramp trajectory of the target 
cursor at time $=+1.47 \mathrm{~s}$ ) and continued throughout the required movement time.

\section{VIBRATION REFLEXES}

In a separate control experiment, vibration reflexes were recorded from three parkinsonian patients (mean age, 62 years, two from main series) and four neurologically unimpaired, control subjects (mean age, 65 years, two from main series). The patients were all at stage II of the Hoehn and Yahr classification ${ }^{20}$. The more affected side was studied in patients and the dominant side was studied in controls.

Subjects voluntarily dorsiflexed the ankle against a force transducer. The leg was supported in the same frame that was used in the main experiment and 2 second trains of Achilles tendon vibration were applied in an identical manner. Immediately before each period of stimulation, subjects were required to attain a dorsiflexor torque of about $15 \%$ of their individual maximum, aided by an oscilloscope display of their contraction strength. They were then asked to close their eyes and to maintain the same level of effort throughout the trial, while not reacting voluntarily to the vibration stimulus. All subjects performed a sequence of 25 trials, each separated by an interval of about 30 seconds, during which subjects relaxed, to allow computation of torque averages.
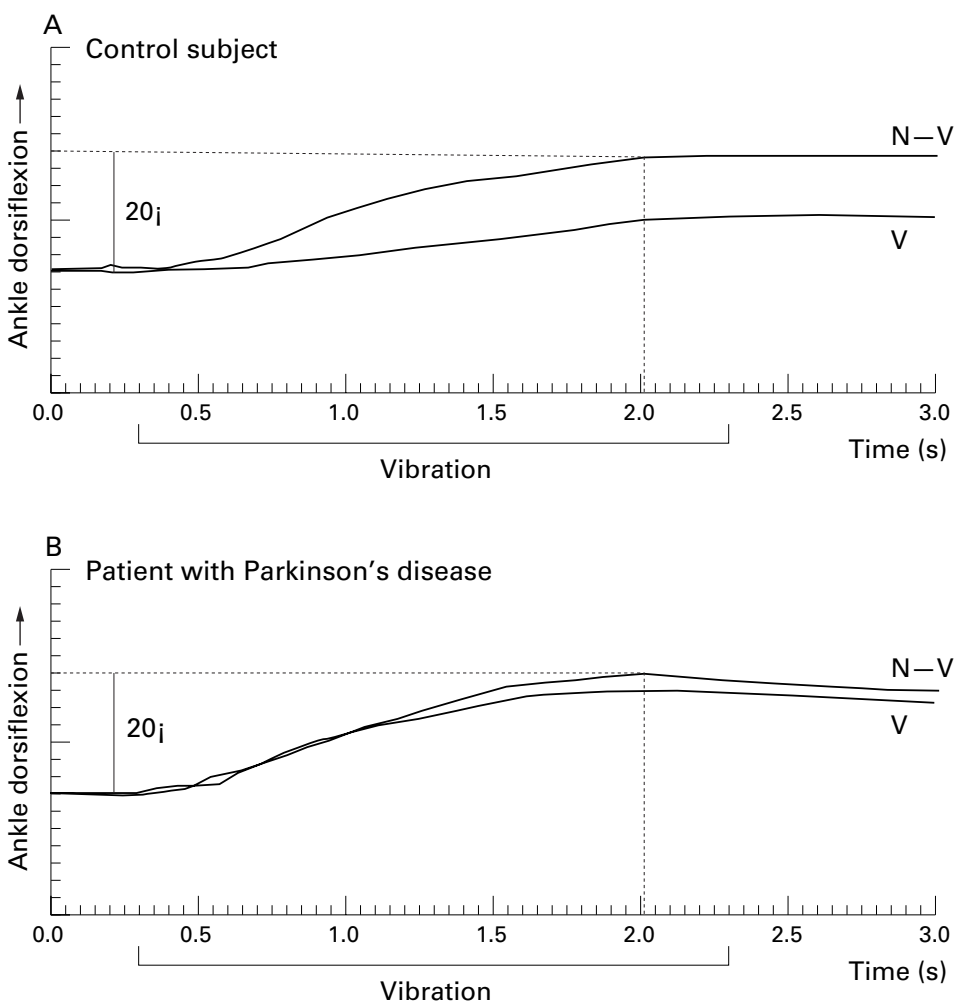

Figure 1 The averaged (20 trials) ankle dorsiflexion trajectories made by a control subject $(A)$ and a patient with Parkinson's disease (B) in the absence (NV) and presence (V) of vibration of the Achilles tendon. The left vertical axis, labelled $0.0 \mathrm{~s}$, corresponds to the timing of the visual "go" cue. The target trajectory was a movement of $20^{\circ}$ (indicated by the vertical calibration bar) in $2.06 \mathrm{~s}$. The measurement time, at $2.0 \mathrm{~s}$ following the "go" cue, is indicated by the vertical dashed line and the period of vibration is indicated by the labelled horizontal bar.
DATA ACQUISITION AND ANALYSES

Movement and stimulus data were analysed off line after tape recorder signal replay, through an I/O interface (CED 1401 plus, CED Ltd, Cambridge, UK), to a personal computer running signal averaging software (SIGAVG, CED Ltd). The end point amplitudes of ankle movements were measured, for each subject, from separately averaged (20 trials) NV and V trajectories, 2 seconds after the visual "go" cue. This measurement time (time $=+3.47 \mathrm{~s}$ relative to auditory warning) preceded the termination of vibration trains by approximately 0.3 seconds.

For each subject, the mean movement amplitude was taken, separately across the $20 \mathrm{~V}$ trials and across the $20 \mathrm{NV}$ trials. The assumptions underlying the use of parametric inferential statistics were not significantly violated in respect of any of the sample distributions of analysed dependent variables. The movementamplitude data were analysed using a 2 (group) $\times 2$ (vibration condition) analysis of variance (ANOVA) using BMDP2V software) with repeated measures on the second factor. Post hoc tests were conducted using, as appropriate, paired or unpaired $t$ tests. The effects of vibration were also expressed in terms of vibrated/non-vibrated (V/NV) ratios, group mean values of which were compared using an unpaired $t$ test. In the patient group, the degree to which V/NV ratios were correlated with age, clinical scores (total Webster ${ }^{21}$ score), or duration of illness was estimated using Pearson's $r$; $\alpha$ was set at 0.05 for all tests.

\section{Results}

VIBRATION INDUCED MOVEMENT ERRORS IN

CONTROL AND PARKINSONIAN SUBJECTS

The application of trains of vibration to the Achilles tendon during practised slow dorsiflexion movements of the ankle induced a marked reduction in the end point amplitude (at measurement time $2.0 \mathrm{~s}$ after the visual "go" cue) of movements made by control subjects. The averaged ankle dorsiflexion movement trajectories made by one such subject in the absence and presence of such vibration of the antagonist muscles are illustrated in fig $1 \mathrm{~A}$. As was found for all control subjects, both the $\mathrm{NV}$ and $\mathrm{V}$ movements made by this person had a fairly smooth, ramp-like profile. The NV movements, on average, closely approximated to the required size $\left(19.45^{\circ}\right.$ at $2.0 \mathrm{~s}$ measurement time). By contrast, the movements made in the presence of antagonist vibration (V) showed a pronounced reduction in movement speed and the target amplitude was undershot by almost $50 \%$. Antagonist vibration elicited a qualitatively similar alteration of movement trajectory in the parkinsonian patients. Examples of the averaged NV and V ankle dorsiflexion movements produced by a patient with Parkinson's disease are presented in fig $1 \mathrm{~B}$. In common with the other members of the parkinsonian group, this patient produced an averaged NV movement with a reasonably uniform trajectory to reach, at 2 seconds, an amplitude which corresponded well with the target end point. Antagonist vibration resulted 


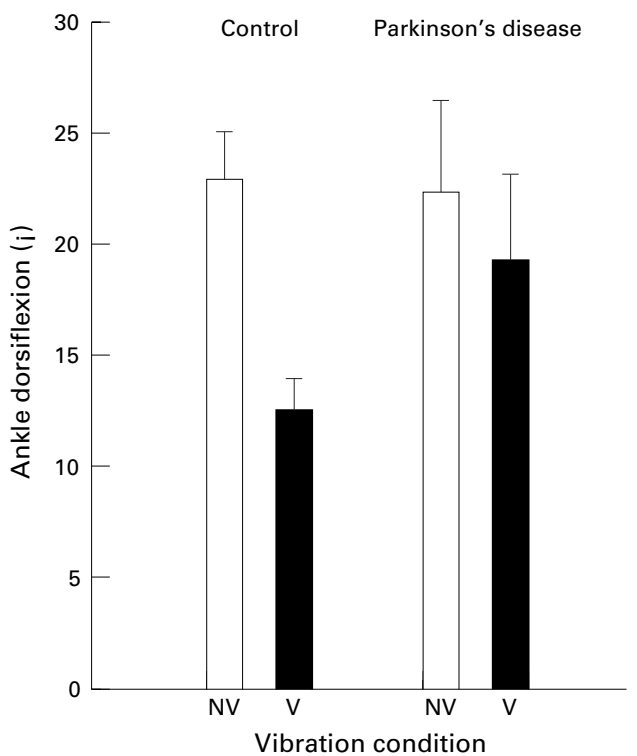

Figure 2 Group mean (+SD) amplitudes of ankle dorsiflexion trajectories made in the absence (NV,open bars) and presence ( $V$, solid bars) of vibration of the Achilles tendon for control subjects and patients with Parkinson's disease.

in a modest, but definite (about 12\%), undershooting of end point amplitude due to a slowing of movement speed.

Analysis of the movement amplitudes at the defined measurement time using a 2 (subject group) $\times 2$ (vibration condition) ANOVA showed significant main effects of group ( $F$ $(1,40)=10.6 ; \mathrm{p}=0.0023)$ and vibration condition $(F(1,40)=751.7 ; \mathrm{p}<0.00005)$, and a significant interaction between the two factors $(F(1,40)=223.5 ; \mathrm{p}<0.00005)$.

The group mean amplitudes for, respectively, NV and V trials produced by control subjects and parkinsonian patients are shown in fig 2. The control group mean (SD) amplitude of averaged NV movements was $22.8(2.2)^{\circ}$ at the standard measurement point, and the corresponding mean value for $\mathrm{NV}$ trials in the patient group was $22.2(4.2)^{\circ}$. The target amplitude was $19.5^{\circ}$. Thus, the sizes of NV movements of both control subjects and patients tended, on average, to exceed slightly the target value. However, the extent of the overshooting was small in both groups and there was no significant between group difference $(t(40)=0.6 ; \mathrm{p}=0.55)$. The mean $(\mathrm{SD})$ amplitudes of $\mathrm{V}$ movements of the ankle for the control and Parkinson's disease groups were, respectively, $12.4(1.5)^{\circ}$ and 19.1 (3.9 $)^{\circ}$. Thus, the amplitudes of $\mathrm{V}$ movements were, on average, about $46 \%$ and $14 \%$ less than their NV counterparts in respectively, the control and patient groups.

Statistical comparison of the NV and V movement amplitudes of the control subjects showed that vibration produced a highly significant reduction in movement size ( $t$ $(20)=25.8 ; p<0.00005)$. Similarly, the V movement amplitudes of the parkinsonian patients were significantly decreased relative to those of corresponding NV trials $(t \quad(20)=11.0$; $\mathrm{p}<0.00005)$. Therefore, antagonist vibration exerted an action of an essentially similar nature in both patients and controls.

The magnitude of the vibration induced reduction in movement amplitude, however, differed between groups. Comparison of the amplitudes of the $\mathrm{V}$ movements produced by the two groups indicated that those of the controls were significantly smaller than those of the patients $(t(40)=7.42 ; \mathrm{p}<0.00005)$, in the absence of a significant between group difference in baseline NV movement amplitudes (see above). This confirms previous findings of a less pronounced movement undershooting in the presence of antagonist vibration in patients with Parkinson's disease than in controls. These results were confirmed by an analysis of V/NV amplitude ratio data, this measure serving as an index of the strength of the vibration effect, normalised with respect to NV movement amplitude. For each subject, a V/NV amplitude ratio was calculated from measurements of the averaged trajectories of $\mathrm{V}$ and $\mathrm{NV}$ trials. Group mean (SD) V/NV ratios for the control and patient groups were, respectively, $0.544(0.060)$ and $0.860(0.055)$. In confirmation of the results of the analyses reported above, the V/NV ratios of the controls were significantly smaller than those of the patients $(t(40)=17.8 ; \mathrm{p}<0.00005)$.

\section{CORRELATION OF V/NV RATIO IN THE PATIENT} GROUP WITH AGE, CLINICAL SIGNS, AND DISEASE DURATION

In the patient group, the $\mathrm{V} / \mathrm{NV}$ ratio (indexing the magnitude of vibration induced undershooting of ankle movements) was not correlated significantly with age $(\mathrm{r}=-0.002$; $\mathrm{p}=0.99)$, disease duration $(r=-0.04 ; \mathrm{p}=0.86)$ nor clinical score $(r=-0.06 ; \mathrm{p}=0.78)$. It should be noted, however, that the 21 patients formed a substantially homogeneous group in respect of the severity of their disease state. Most patients had Webster's ${ }^{21}$ ratings around 10, and all but one were assigned a Hoehn and Yahr ${ }^{20}$ staging of II (table).

\section{VIBRATION REFLEXES}

To assess the possible contribution of vibration reflexes to trajectory errors, recordings were made, under isometric conditions, of the changes in ankle torque that accompanied 2 seconds trains of mechanical stimulation of the Achilles tendon of the essentially relaxed triceps surae while subjects attempted to maintain a steady level of voluntary dorsiflexor effort of $15 \%$ maximum.

Comparison of the torque measurements from averaged ( 25 trials) traces at the onset and termination of vibration indicated that in each of the subjects tested (three parkinsonian patients and four controls), reductions in ankle dorsiflexor force occurred during stimulation. However, considering all subjects, these reductions in net torque were modest and ranged between 3 and $8 \%$ of prestimulus levels. There was considerable overlap in the fractional changes in the two groups: the percentage reductions in dorsiflexor torque for the three patients were $5 \%, 6 \%$, and $6 \%$, and the corresponding values for the four controls were $3 \%$, 
$5 \%, 7 \%$, and $8 \%$. Two of the controls and two of the patients had participated in the main experiment. The decreases in isometric torque shown by these two control subjects were 5\% and $7 \%$ and their $\mathrm{V} / \mathrm{NV}$ ratios were, respectively, 0.53 and 0.57 . Both of these patients with Parkinson's disease gave 6\% depressions of isometric torque and their $\mathrm{V} / \mathrm{NV}$ ratios were 0.84 and 0.92 . Thus, the $\mathrm{V} / \mathrm{NV}$ ratios of those subjects who participated in both tasks were within the midranges for the broader control and Parkinson's disease groups. The finding that isometric torque consistently returned to prestimulus levels within a few hundred $\mathrm{ms}$ of cessation of vibration militates against the force changes having been caused by an underlying, sustained reduction in the subjects' voluntary drive during trials. Therefore, these results suggest that vibration did exert reflex actions which tended to reduce dorsiflexor contraction and which seem likely to have contributed to movement undershooting in the main experiment. Such effects, however, appeared to be relatively weak and not to differ systematically between the patients and controls.

\section{Discussion}

The principal novel finding in our experiments was that antagonist (plantarflexor) muscle vibration evoked a significantly smaller degree of undershooting of slow, practised, voluntary dorsiflexor movements of the ankle in patients with idiopathic Parkinson's disease than in age matched, neurologically unimpaired controls. However, the finding that vibration induced qualitatively similar alterations of movement trajectories (curtailment of movement extent) in each subject group suggests that the reduced effect of stimulation in parkinsonian patients reflects a subtle impairment of normal processes rather than the appearance of a new pathological process. The results reported here, allied to earlier analogous data for wrist movements, ${ }^{17}$ strengthen the conclusion that a disturbance of proprioceptive guidance may be a generalised feature of Parkinson's disease.

NORMAL MECHANISMS OF VIBRATION INDUCED MOVEMENT ERRORS AND THEIR DISTURBANCE IN PARKINSON'S DISEASE

It is well established that, in healthy subjects, muscle vibration has both sensory and motor consequences. The first includes distortions of movement and position senses, ${ }^{4}$ and the second includes the generation of reflexes ${ }^{22}$ and modifications of the trajectories of willed movements. ${ }^{6} 723$

The possible mechanisms responsible for the undershooting of voluntary movements which typically accompanies antagonist muscle vibration in normal subjects have been thoroughly discussed. $^{7917}$ These include the actions of vibration reflexes and/or voluntary "corrective" motor responses to perceived errors of movement speed. The prevailing view is that an alteration in volitional drive, secondary to a vibration induced illusion of movement, is the dominant factor. ${ }^{17}$ This opinion is supported by the results of our second experiment, which was performed under isometric conditions to minimise changes in voluntary commands. In this situation, the (presumed reflex) reductions (5\%-6\%, controls, and patients) in pre-existing net force were too small to explain the pronounced undershooting of voluntary ankle dorsiflexion movements found in the main experiment (about $45 \%$ and $15 \%$, respectively, in control and patient groups).

The extent of antagonist vibration induced undershooting of the dorsiflexion ankle movements made by our controls (group mean $\mathrm{N} / \mathrm{NV}$ ratio, 0.54 ) was of a similar order to that reported previously for wrist movements (group mean ratio, 0.66). ${ }^{17}$ Thus, willed ankle movements were not immune to disruption by experimental stimulation of proprioceptors, despite the ankle musculature being usually associated with more automatic activities ${ }^{24}$ and being considered to be under somewhat less secure cortical control.

The reduced vibration induced errors found in our patients with Parkinson's disease might potentially result from disturbances of peripheral (for example, diminished proprioceptor responsiveness), lower central (for example, abnormal reflex actions) and/or higher central (for example, defective comparator function or impaired cognitive or attentional capabilities) mechanisms.

The main class of sensory receptor that is generally thought to be involved in vibration illusions and reflexes is the muscle spindle and, in particular, the spindle primary ending. ${ }^{4}$ However, decreased spindle stimulation in parkinsonian patients is improbable. The application of vibration was highly standardised, the effectiveness of stimulus spread into the triceps surae muscles was routinely confirmed by palpation and small, autogenetic vibration reflexes, comparable with those elicited in controls, were regularly found. Furthermore, human microneurographic recordings in patients with Parkinson's disease have indicated that vibration evokes patterns of spindle afferent firing which are basically similar to those found in healthy subjects. ${ }^{25}$

There was no indication, moreover, that an abnormality of vibration reflexes was responsible for the reduction in vibration induced undershooting of target trajectories shown by our patients. A reflex explanation of such reduced undershooting would require either (1) an exaggeration of autogenetic excitatory reflexes in the vibrated triceps surae, exerting a braking action and/or (2) a depression of reciprocal inhibition of the prime mover tibialis anterior in parkinsonian patients. As noted earlier, however, mechanical recordings showed that the (presumed reflex) reductions in dorsiflexor force evoked under isometric conditions by prolonged trains of vibration were modest in both controls and patients. More important, in the present context, was the finding that there was no obvious difference in behaviour between the two groups. The results of several earlier studies also militate against a reflex explanation. Phasic and tonic excitatory vibration reflexes have not been found to be depressed in patients with Parkinson's disease; rather, they have been reported 
to be normal ${ }^{15}{ }^{17}$ or augmented. ${ }^{26}$ In addition, the evidence concerning any possible decrease in reciprocal inhibition in parkinsonian patients is equivocal. Lelli et $a l^{27}$ have reported, using $\mathrm{H}$ reflex methodology, that early and late components of reciprocal inhibition are depressed in the upper limb muscles of patients with Parkinson's disease. More recently, however, Nakashima et $a l^{28}$ have described the earlier phases of reciprocal inhibition as being normal, and the late phase as enhanced, in the forearm musculature of parkinsonian patients whereas short latency reciprocal inhibition has been found to be increased in the leg muscles of patients with Parkinson's disease. ${ }^{29}$ By elimination, a disturbance of some aspect of higher level proprioceptive function is the most likely reason for the failure of parkinsonian patients to respond to a normal extent to experimentally evoked muscle spindle input.

Traditionally, the control of slow, nonballistic movements is thought to be mediated by a negative feedback system involving the comparison of a motor command signal, possibly in the form of corollary discharges, of the required trajectory with a sensory feedback signal of the actual evolving movement trajectory. ${ }^{30}$ According to this type of control model, the reduced vibration induced undershooting shown by parkinsonian patients would result from either an attenuation or saturation of the proprioceptive feedback signal, or the feedback signal receiving a low weighting within the comparator. Support for the presence of impaired central processing of proprioceptive input by the basal ganglia in patients with Parkinson's disease is provided by single unit recordings in the MPTP treated primate. For instance, pallidal neurons of parkinsonian monkeys show altered resting discharge rates, reduced selectivity, and greatly enhanced responses to peripheral inputs. ${ }^{31} 32$ Such an exaggeration of neuronal responsiveness within the basal ganglia would, at first sight, seem to be inconsistent with the notion that Parkinson's disease involves an attenuation of sensory feedback. However, it is possible that a saturation or ceiling effect may operate so that pronounced increases in afferent firing (for example, by vibration) would provoke relatively little change in the signal to the comparator. Thus, it is plausible that saturation of sensory feedback within the diseased basal ganglia could contribute to abnormal proprioceptive guidance of movements in parkinsonian patients.

A related explanation concerns possible deficits of cognitive functions in patients with Parkinson's disease. Our finding that the parkinsonian patients made abnormally small vibration evoked adjustments to trajectories implies that either the patients experienced the accompanying illusions to a lesser extent than controls or failed to modify ongoing motor programmes on the basis of their movement percepts. To date, in only one study ${ }^{33}$ has an evaluation of vibration induced kinaesthetic illusions been made in parkinsonian patients using a reporting method which did not itself require subjects to make active movements of the test arm (instead, subjects set the speed of a moving visual display to match their perceived limb motion). In these experiments, Moore $^{33}$ found no definite disturbances in the small sample of patients that he examined. Clear impairments of somatosensory perception in patients with Parkinson's disease have been demonstrated, however, in various studies of, for example, tactile discrimination ${ }^{34-36}$ and elements of joint position sense. ${ }^{37-39}$

RELEVANCE OF OUR FINDINGS TO MOTOR FUNCTION IN PATIENTS WITH PARKINSON'S DISEASE

Our experimental results, in conjunction with earlier similar results derived from wrist movements, ${ }^{17}$ suggest strongly that a disruption of proprioceptive regulation is characteristic of willed movement in Parkinson's disease. The extent to which such a disturbance of proprioceptive guidance contributes to patients' motor impairments is unclear. Potentially, however, several components of the ensemble of movement features encompassed by the bradykinetic syndrome might originate from inadequate sensory modulation or reinforcement of motor drive. The lower movement speed of the more affected limb, during attempted matching of bilateral trajectories by parkinsonian patients with asymmetric disease, ${ }^{16}$ may reflect a failure to make sufficiently large compensatory adjustments to inequalities of proprioceptive discharge from the two sides. Similarly, the reduced size of parkinsonian movements in other contexts - for example, steps during locomotion and pen strokes during writingmay result partly from an inability to scale motor activity appropriately according to proprioceptive cues. The work was supported by The Wellcome Trust and carried
out in the Institute of Clinical Physiology, Manchester Royal Infirmary. Mr S Hacks assisted with data collection for the Infirmary. Mr S Hacks assi
vibration reflex experiments.

1 Mott FW, Sherrington CS. Experiments upon the influences of sensory nerves upon movement and nutrition of the limbs. Proc Royal Soc Lond B Biol Sci 1895;57:481-8.

2 Foerster, O. Schlaffe und spastiche Lahmung. In: Berthe A, v Bergman G, Embden G, et al, eds. Handbuch der normalen und pathologischen physiologie. Vol 10. Berlin: Springer, 1927:900-1

3 Rothwell JC, Traub MM, Obeso JA, et al. Manual performance in a de-afferented man. Brain 1982;105:515-42.

4 Goodwin GM, McCloskey DI, Matthews PBC. The contribution of muscle afferents to kinaesthesia shown by vibration-induced illusions of movement and the effects of paralyzing joint afferents. Brain 1972;95:705-48.

5 Gilhodes JC, Roll JP, Tardy-Gervet MF. Perceptual and motor effects of agonist-antagonist muscle vibration in man. Exp Brain Res 1986;61:395-402.

6 Capaday C, Cooke JD. The effects of muscle vibration on the attainment of intended final position during voluntary the attainment of intended final position during

7 Capaday C, Cooke JD. Vibration-induced changes in movement-related EMG activity in humans. Exp Brain Res movement-related

8 Lackner JR. Some influences of tonic vibration reflexes on position sense of the contralateral limb. Exp Neurol 1984;85:107-13.

9 Sittag AC, Dernier van der Gon JJ, Gielen CCAM. Separate control of arm position and velocity demonstrated by vibration of muscle tendon in man. Exp Brain Res 1984;60: 445-53

10 Dinnerstein AJ, Frigysei T, Lowenthal M. Delayed feedback as a possible mechanism in parkinsonism. Percept Mot Skills 1962;15:667-80.

11 Schneider JS, Denaro FJ, Lidsky TI. Basal ganglia: motor influences mediated by sensory interactions. Exp Neurol

12 Brown LL, Schneider JS, Lidsky TI. Sensory and cognitive functions of the basal ganglia. Curr Opin Neurobiol 1997;7: 157-63. 
13 Tatton WG, Lee RG. Evidence for abnormal long-loop reflexes in rigid parkinsonian patients. Brain Res 1975;100 reflexes

14 Mortimer JA, Webster DD. Evidence for a quantitative association between EMG stretch responses and parkinsonian rigidity. Brain Res 1979;162:169-73.

15 Cody FWJ, MacDermott N, Matthews PBC, et al. Observations on the genesis of the stretch reflex in Parkinson's disease. Brain 1986;109:229-49.

16 Moore AP. Impaired sensorimotor integration in parkinsonism and dyskinesia: a role for corollary discharges. 7 Neuro Neurosurg Psychiatry 1987;50:544-52.

17 Rickards C, Cody FWJ. Proprioceptive control of wrist movements in Parkinson's disease: reduced muscle vibration-induced errors. Brain 1997;120:977-90.

18 Khudados E, Cody FWJ, O'Boyle DJ. Vibration-induced errors of human voluntary ankle movements are reduced in
Parkinson's disease. F Physiol (Lond) 1997;501.P:44P.

19 Hughes AJ, Daniel SE, Kilford L, et al. Accuracy of clinical diagnosis of idiopathic Parkinson's disease: a clinicodiagnosis of idiopathic Parkinson's disease: a clinicotry 1992;55:181-4, 938-9.

20 Hoehn MM, Yahr MD. Parkinsonism: onset, progression and mortality. Neurology 1967;17:427-42.

21 Webster DD. Critical analysis of the disability in Parkinson's disease. Mod Treatment 1968;5:257-82.

22 Hagbarth K-E, Eklund G. Motor effects of vibratory muscle stimuli in man. In: Granit R, ed. Muscular afferents and motor control. Stockholm: Almqvist and Wiksell, 1966:17786.

23 Cody FWJ, Schwartz MP, Smit G. Proprioceptive guidance of human voluntary wrist movements studied using muscle vibration. $\mathcal{F}$ Physiol (Lond) 1990;427:455-70.

24 Jackson JH. In: Taylor J, ed. Selected writings of fohn Hughlings fackson. London: Hodder and Stoughton, 1932, 427.

25 Burke D, Hagbarth K-E, Wallin BG. Reflex mechanisms in parkinsonian rigidity. Scand F Rehab Med 1977;9:15-23.

26 McLellan DL. Clinical and neurophysiological aspects of extrapyramidal disease in man [PhD thesis]. Glasgow: University of Glasgow, 1975:167-82.
27 Lelli S, Panizza M, Hallett $M$. Spinal cord inhibitory mechanisms. Neurology 1991;41:553-6.

28 Nakashima K, Shimoyama R, Yokoyama R, et al. Reciprocal inhibition between forearm muscles in patients with Parkinson's disease. Electromygr Clin Neurophysiol 1994;34: 67-72.

29 Delwaide PJ, Pepin JL, Maertens de Noordhout A. Short-latency autogenic inhibition in patients with Parkinsonian rigidity. Ann Neurol 1991;30:83-9.

30 Matthews PBC. Where does Sherrington's muscular sense originate? Muscles, joints, corollary discharges?. Anпи Rev Neurosci 1982;5:189-218.

31 Tremblay L, Filion M, Bedard PJ. Responses of pallidal neurons to striatal stimulation in monkeys with MPTPinduced parkinsonism. Brain Res 1989;498:17-33.

32 Filion M, Tremblay L. Abnormal spontaneous activity of globus pallidus neurons in monkeys with MPTP-induced parkinsonism. Brain Res 1991;547:142-51.

33 Moore AP. Vibration-induced illusions of movement are normal in Parkinson's disease: implications for the mechanisms of the movement disorder. In: Crossman AR, Sambrook MA, eds. Neural mechanisms in disorders of moveSambrook MA, eds. Neural mechanisms in

34 Schneider JS, Diamond SG, Markham CH. Deficits of orofacial sensorimotor function in Parkinson's disease. Ann Neurol 1986;19:275-82.

35 Sathian K, Zangaladze A, Green J, et al. Tactile spatial acuity and roughness discrimination: impairments due to aging and Parkinson's disease. Neurology 1997;49:168-77.

36 Zia S, Cody FWJ, O'Boyle DJ. Impairment of discrimination of bilateral differences in the loci of tactile stimuli in Parkinson's disease. F Physiol (Lond) 1998;509.P:180-1P.

37 Zia S, Cody FWJ. Evidence of impairment of human joint position sense in

38 Zia S, Cody FWJ, O'Boyle DJ. Impaired joint position sense in Parkinson's disease during active maintenance of joint in Parkinson's disease during active maint
angle. F Physiol (Lond) 1996;494.P:68P.

39 Zia S, Cody FWJ, O'Boyle DJ. Disturbance of human joint position sense in Parkinson's disease. $f$ Physiol (Lond) position sense in Park 